\title{
Assessment of Leptomeningeal Carcinomatosis Diagnosis, Management and Outcomes in Patients with Solid Tumors Over a Decade of Experience
}

(1) Hannah Rinehardt', (1) Mahmoud Kassem²,3, (1) Evan Morgan ${ }^{2,3}$, (1) Marilly Palettas ${ }^{4}$, (1) Julie A. Stephens $s^{4}$,

(1) Anupama Suresh ${ }^{2,3}$, (1) Akansha Ganju $u^{2,3}$, (1) Maryam Lustberg, ${ }^{2,3}$, (1) Robert Wesolowski ${ }^{2,3}$, (1) Sagar Sardesai ${ }^{2,3}$,

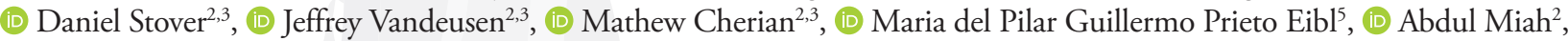

(1) Iyad Alnahhas5, (1) Pierre Giglio5, (1) Vinay K. Puduvalli, (1) Bhuvaneswari Ramaswamy ${ }^{2,3}$, (1) Nicole Williams ${ }^{2,3}$,

(D) Anne M. Noonan ${ }^{2}$

${ }^{1}$ The Ohio State University College of Medicine, Columbus, OH, USA

${ }^{2}$ Division of Medical Oncology, Comprehensive Cancer Center, The Ohio State University Wexner Medical Center, Columbus, OH, USA

${ }^{3}$ Stefanie Spielman Comprehensive Breast Cancer, The Ohio State University, Columbus, OH, USA.

${ }^{4}$ Center for Biostatistics, Department of Biomedical Informatics, The Ohio State University College of Medicine Columbus, OH, USA

${ }^{5}$ Division of Neuro-oncology, The Ohio State University Wexner Medical Center, Columbus, OH, USA

\begin{abstract}
Objective: Leptomeningeal carcinomatosis (LMC), a common complication of advanced malignancies, is associated with high morbidity and mortality, yet diagnosis and treatment decisions remain challenging. This study describes the diagnostic and treatment modalities for LMC and identifies factors associated with overall survival (OS).

Materials and Methods: We performed a single-institution retrospective study (registration \#: OSU2016C0053) of 153 patients diagnosed with LMC treated at The Ohio State University, Comprehensive Cancer Center, (OSUCCC)-James between January 1, 2010 and December 31, 2015.

Results: Median age at diagnosis was 55.7 years, and $61 \%$ had Eastern Cooperative Oncology Group baseline performance status $\leq 1$. Most common primary tumors were breast $(43 \%)$, lung $(26 \%)$, and cutaneous melanoma (10\%). At presentation, most patients were stage III-IV $(71 \%)$ with higher grade tumors (grade III: $46 \%)$. Metastases to bone (36\%), brain (33\%), and lung (12\%) were the most common sites with a median of 0.5 years (range, $0-14.9$ years) between the diagnosis of first metastasis and of LMC. 153 (100\%) patients had MRI evidence of LMC. Of the 67 (44\%) who underwent lumbar puncture (LP), 33 (22\%) had positive cerebrospinal fluid (CSF) cytology. Most patients received radiotherapy for LMC (60\%) and chemotherapy (93\%) for either the primary disease or LMC. 28 patients received intrathecal chemotherapy, 22 of whom had a primary diagnosis of breast cancer. $98 \%$ died with median OS of all patients was 1.9 months (95\% CI: 1.3-2.5 months).
\end{abstract}

Conclusion: Despite improved treatments and targeted therapies, outcomes of LMC remain extremely poor. Positive CSF cytology was associated with lower OS in patients who had cytology assessed and specifically in patients with breast cancer. CSF cytology serves as an important indicator for prognosis and helps aid in developing individualized therapeutic strategies for patients with LMC.

Keywords: Leptomeningeal carcinomatosis, breast cancer, metastasis, cerebrospinal fluid, magnetic resonance imaging

Cite this article as: Rinehardt H, Kassem M, Morgan E, Palettas M, Stephens JA, Suresh A, Ganju A, Lustberg M, Wesolowski R, Sardesai S, Stover D, Vandeusen J, Cherian M, del Pillar Guillermo M, Eibl P, Miah A, Alnahhas I, Giglio P, Puduvalli VK, Ramaswamy B, Williams N, Noonan AM. Assessment of Leptomeningeal Carcinomatosis Diagnosis, Management and Outcomes in Patients with Solid Tumors Over a Decade of Experience.

Eur J Breast Health 2021; 17(4): 371-377.

\section{Key Points}

- $\quad$ LMC most commonly presents with late-stage cancers with cancers of the breast, lung and melanoma being the most common primary cancers.

- Diagnosis of LMC may be challenging and imaging with MRI brain and spine was most frequently used in our study as an aid in diagnosis and in some cases as the primary tool for diagnosis.

- CSF cytology is the gold standard for diagnosis but is not always technically possible to obtain as demonstrated by only 67 of 153 patients in this review having CSF sampled. 
- Prognosis was worse in patients with positive CSF cytology versus equivocal or negative cytology.

- Treatment of LMC either by intrathecal chemotherapy, radiation to the brain or spine, or systemic therapy was associated with an improvement in survival versus no treatment.

\section{Introduction}

Leptomeningeal carcinomatosis (LMC) is defined as metastatic involvement of the leptomeninges, subarachnoid space and cerebrospinal fluid (CSF) (1). Malignant tumor cells spread and disseminate to the subarachnoid space by hematogenous, perineural, lymphatic, or perivascular mechanisms or by direct extension from superficial brain metastases or bone metastases of the calvarium or spine (2-4). The incidence of LMC is increasing as patient survival improves with advances in the management of metastatic solid tumors and as magnetic resonance imaging (MRI) becomes more widely utilized $(5,6)$. LMC occurs in approximately $4 \%-15 \%$ of patients with malignant solid tumors, most commonly melanomas and malignancies of the breast, lung, and gastrointestinal organs (7-11). Signs and symptoms of LMC include headaches, vomiting, seizures, focal neurologic deficits, radicular neck and back pain, cerebellar dysfunction, altered mental status, cauda equina syndrome, dizziness, or syncope (12-14). The sensitivity and specificity of MRI in the diagnosis of LMC is difficult to estimate due to poor concordance with the gold standard diagnostic test of positive CSF cytology $(15,16)$. MRI with and without contrast is the initial and often the sole diagnostic tool for LMC (17). Definitive diagnosis of LMC depends on the presence of malignant cells in the CSF, but sensitivity is limited at about $50 \%-60 \%$ for the first lumbar puncture (LP) $(6,18,19)$. If the first CSF analysis is negative, a second LP can increase sensitivity to $80 \%-85 \%$ (20). As a result of low sensitivity and patient intolerance one or more LPs, a probable diagnosis of LMC is made when MRI findings are present in the setting of systemic malignancy, even in the absence of positive CSF cytology (16).

Once diagnosed with probable or definitive LMC, median survival time for patients is 2-6 months with treatment (21-25). Most treatment recommendations are based on clinical experience or studies with a low level of evidence due to a lack of prospective, randomized trials for patients with LMC (26). Intrathecal chemotherapy is the direct instillation of chemotherapy into the subarachnoid space, making it a promising treatment strategy. Intra-CSF pharmacotherapy should be reserved mainly for patients with a positive cytology on LP given that clearance of CSF cytology is used as one indicator for efficacy of this treatment (27). This is usually provided via an Ommaya reservoir after adequate CSF flow is confirmed using ${ }^{111}$ Indium-DTPA flow study.

Whole Brain Radiotherapy (WBRT) with whole spine irradiation can target the entire craniospinal axis and thus a larger area of disease burden in LMC, however its use is limited by significant myelotoxicity (26). Focal external beam radiation to areas of bulky leptomeningeal involvement of the spine causing CSF obstruction can be utilized to relieve symptoms and allow for the administration of intrathecal administration $(26,28)$. The survival benefit of the various radiation therapy modalities in LMC is unclear.

We conducted a retrospective study to assess the diagnosis, management and outcomes of leptomeningeal carcinomatosis at The

\section{Materials and Methods}

\section{Study design and data collection}

This study was an IRB-approved (registration \#: OSU2016C0053) retrospective chart review of clinical and histopathologic data from patients treated at The Ohio State University Comprehensive Cancer Center, (OSUCCC)-James that was initially approved on 05/04/2016 between January $1^{\text {st }}, 2010$ and December 31 2015. Eligible patients were identified by ICD-9 and ICD-10 codes (198.4/ C79.32, C79.49, respectively) and included patients who were diagnosed with leptomeningeal carcinomatosis or unspecified meningeal disease, as well as patients who were diagnosed with a malignant solid tumor, who had undergone a procedure indicative of leptomeningeal carcinomatosis according to current procedural terminology (CPT) codes. These procedures included insertion of cerebrospinal fluid drainage device or catheter, LP, intrathecal infusion or injection of a therapeutic or prophylactic substance, injection or infusion of cancer chemotherapeutic substance with destruction of blood brain barrier, or MRI imaging of the brain or spinal cord. Patients without LMC, patients with LMC secondary to leukemia, lymphoma, or primary central nervous system malignancies, patients with incomplete clinical data and those treated at other institutions were excluded. Per EANO-ESMO Clinical Practice Guidelines, MRI is the gold standard imaging tool for imaging suspected cases of LMC. Given the technical challenges of doing a lumbar puncture on some poor performance patients, we defined a case of LMC as having either positive CSF cytology or MRI imaging indicative of LMC. Of 469 medical records reviewed, 153 patients were determined eligible.

Data for the eligible patients were initially obtained from The Ohio State University Information Warehouse and uploaded into REDCap (29). Data missing from the initial query were populated using manual review of each patient's electronic medical record.

\section{Outcome measures}

The primary objectives of this study were to assess the overall survival (OS) of patients with LMC at the OSU-CCC James, and to examine if primary tumor characteristics, diagnostic information, management modalities (locoregional, systemic, or combined therapy) and demographic factors were associated with OS. We performed a specific subgroup analysis to assess treatment strategies and outcomes among LMC patients with primary breast cancer overall and each histologic subtype of breast cancer including hormone receptor positivity.

A change in treatment after LMC diagnosis was defined as a patient receiving any of the following new treatments or changes in initial therapy: focal radiation therapy to brain metastases, bulky sites of LMC burden or whole brain radiation therapy (WBRT); initiating IT chemotherapy, discontinuing previous systemic therapy, or initiating new systemic therapy. If a patient did not undergo any of the previously mentioned changes, they were considered as having no new treatment, even if continuing with any previous systemic therapy treatments or opting for supportive care alone. 


\section{Statistical analysis}

Demographic and clinical characteristics were summarized using descriptive analysis reported as medians and interquartile range for continuous variables and frequencies and percents for categorical variables. Overall survival (OS) was defined as the time from the date of diagnosis to date of death due to any cause or last known follow-up. Patients were censored at the date last known to be alive. OS estimates were generated using Kaplan-Meier methods and compared using log-rank tests. All data analyses were performed using SAS 9.4 (SAS Institute Inc., Cary, NC) or Stata 14 (StataCorp LLC, College Station, TX). For comparison of continuous data of one variable between two groups, student's t-test was used. A two-sided p-value of $<0.05$ was considered significant in all analyses.

\section{Results}

\section{Demographic features and clinical findings}

A summary of demographic and clinical characteristics of eligible patients is displayed in Table 1. Eligible patients were predominately Caucasian (84\%), with a median age at LMC diagnosis of 55.7 years (range: $25.0-84.9$ years). The most common sites of primary tumor were breast, lung, and melanoma (43\%, 26\%, and 10\%, respectively). Tumors associated with LMC were characterized by high grade histology (3\% grade 1, 18\% grade 2, and $46 \%$ grade 3 ), advanced stage disease at presentation (Stage I 7\%, Stage II 17\%, Stage III $25 \%$, and Stage IV 46\%), and nodal involvement (71\%). In patients with metastases prior to LMC diagnosis, the most common sites were bone (36\%), brain (33\%), and lung (12\%). The baseline Eastern Cooperative Oncology Group (ECOG) performance status at the time diagnosis of $\mathrm{LMC}$ was $<1$ in $61 \% \geq 2$ in $35 \%$ of patients.

\section{Outcomes}

Among this cohort, there were 150 (98\%) observed deaths. The median OS was 1.9 months [ $95 \%$ confidence interval (CI): 1.3, 2.5]. The median time from primary cancer diagnosis to development of LMC was 2 years [interquartile range (IQR): $1-5.4$ years]. The median time from initial metastatic disease to development of LMC was 0.5 years (IQR: 0-1.9 years) overall and was similar among primary cancer subtypes. Breast cancer was associated with the longest interval from metastasis to LMC of 0.7 years (IQR: 0.0-2.4 years), and lung cancer was associated with the shortest interval of 0.5 years (IQR: $0.0-0.8$ years).

Differences were noted in the Kaplan-Meier estimates for OS between primary cancer diagnoses. The median OS in primary breast cancer was 2.4 months $(95 \% \mathrm{CI}: 1.2,4.4)$, primary lung cancer was 1.3 months (95\% CI: 0.9, 2.1), primary melanoma was 1.7 months $(95 \%$ CI: $0.8,3.5)$, and other primary cancers was 2.6 months (95\% CI: $0.7,3.5$ ) (analysis of variance $\mathrm{p}=0.012$ ) (Figure 1 ). There was no difference detected in OS between ECOG performance status groups with a median OS of 2.0 months $(95 \%$ CI: 1.5, 2.8) for patients with ECOG performance status $0-2$ and 0.7 months $(95 \%$ CI: $0.5,3.2)$ for those with ECOG performance status 3 or $4(\mathrm{p}=0.255)$.

\section{Diagnostic findings}

MRI of the brain and/or spine was performed in all patients (100\%), and of those, $97 \%$ of patients had radiographic evidence of LMC. Figure $2(a, b)$ shows an example of an MRI brain and lumbar spine with leptomeningeal enhancement consistent with LMC. Of the 67 patients who underwent LP, CSF cytology was positive in 49\%,
Table 1. Demographic Summary

Total $(n=153)$

$\begin{array}{llc}\begin{array}{l}\text { Age at LMC } \\ \text { diagnosis }\end{array} & \text { Median [IQR] } & 55.7 \text { years }[48,62.5] \\ & \text { White } & 128(84 \%) \\ \text { Race } & \text { Black } & 16(10 \%) \\ & \text { Other } & 9(6 \%)\end{array}$

Site of primary cancer diagnosis

Breast

$66(43 \%)$

Lung

$40(26 \%)$

Melanoma

$16(10 \%)$

Head/Neck

$8(5 \%)$

Renal

Ovarian

3 (2\%)

Prostate

$4(3 \%)$

Other

$14(9 \%)$

Initial stage at diagnosis

I

$10(7 \%)$

II

$26(17 \%)$

III

$38(25 \%)$

IV

71 (46\%)

Unknown

8 (5\%)

Histologic Grade

I

II

$28(18 \%)$

III

$71(46 \%)$

Unknown

$50(33 \%)$

Biomarker status for breast primary ( $n=66$ )

Estrogen receptor

Negative

$24(16 \%)$

Positive

$40(26 \%)$

Unknown

Progesterone receptor

Negative

$30(20 \%)$

Positive

$30(20 \%)$

Unknown

$3(2 \%)$

HER2 status

Negative

$45(29 \%)$

Equivocal

Positive

$18(12 \%)$

Unknown

$3(2 \%)$

Nodal involvement

Yes

109 (71\%)

No

Unknown

$8(5 \%)$ 
Table 1. Continued

Total $(n=153)$

\section{ECOG performance status}

0

$31(20 \%)$

1

2

$62(41 \%)$

$33(22 \%)$

$17(11 \%)$

$3(2 \%)$

7 (5\%)

Unknown

$55(36 \%)$

Bone

Brain

$18(12 \%)$

Lung

Spinal Cord

Other

$17(11 \%)$

None

Missing

LMC: Leptomeningeal carcinomatosis, IQR: Interquartile range, HER2: Human epidermal growth factor receptor 2, CSF: cerebrospinal fluid, ECOG: Eastern Cooperative Oncology Group, n: Number

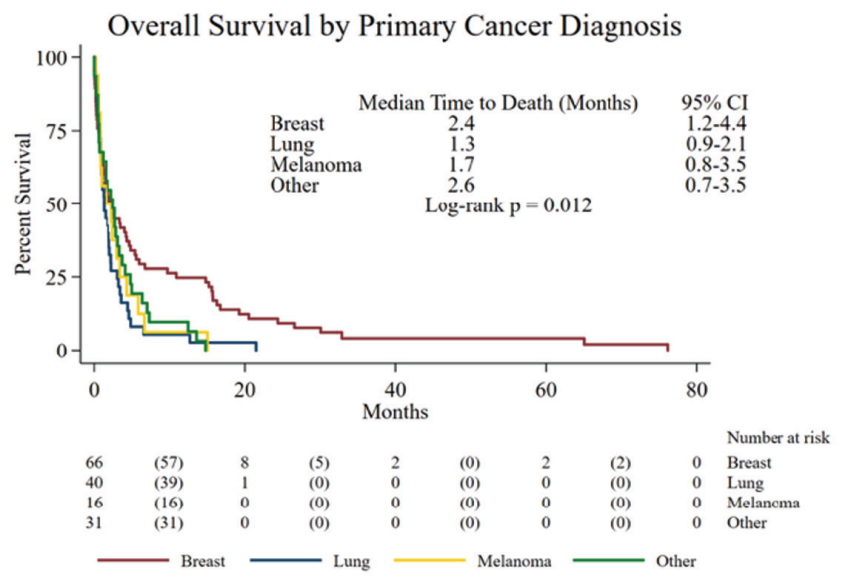

Figure 1. Kaplan-Meier survial curves showing the overall survival for patients with LMC secondary to breast cancer, lung cancer, melanoma, and other tumors

equivocal (suspicious or atypical cells present) in 15\%, and negative in $36 \%$. Figure 3 shows an example of CSF cytology showing LMC from a patient with poorly differentiated gastric carcinoma with signet ring features. As depicted in Figure 4, the Kaplan-Meier curves revealed differences in OS by CSF cytology: median OS for CSF negative patients was 3.8 months (95\% CI: 2.1, 9.8), for CSF equivocal was 2.4 months (95\% CI: $0.5,11.0)$, and for CSF positive patients was 0.9 months (95\% CI: 0.5, 1.3) $(\mathrm{p}<0.005)$.

\section{Management of therapeutic strategy}

Of the 153 patients, 24 (16\%) had no new treatment after LMC diagnosis and 129 (84\%) had a new addition of radiation to the
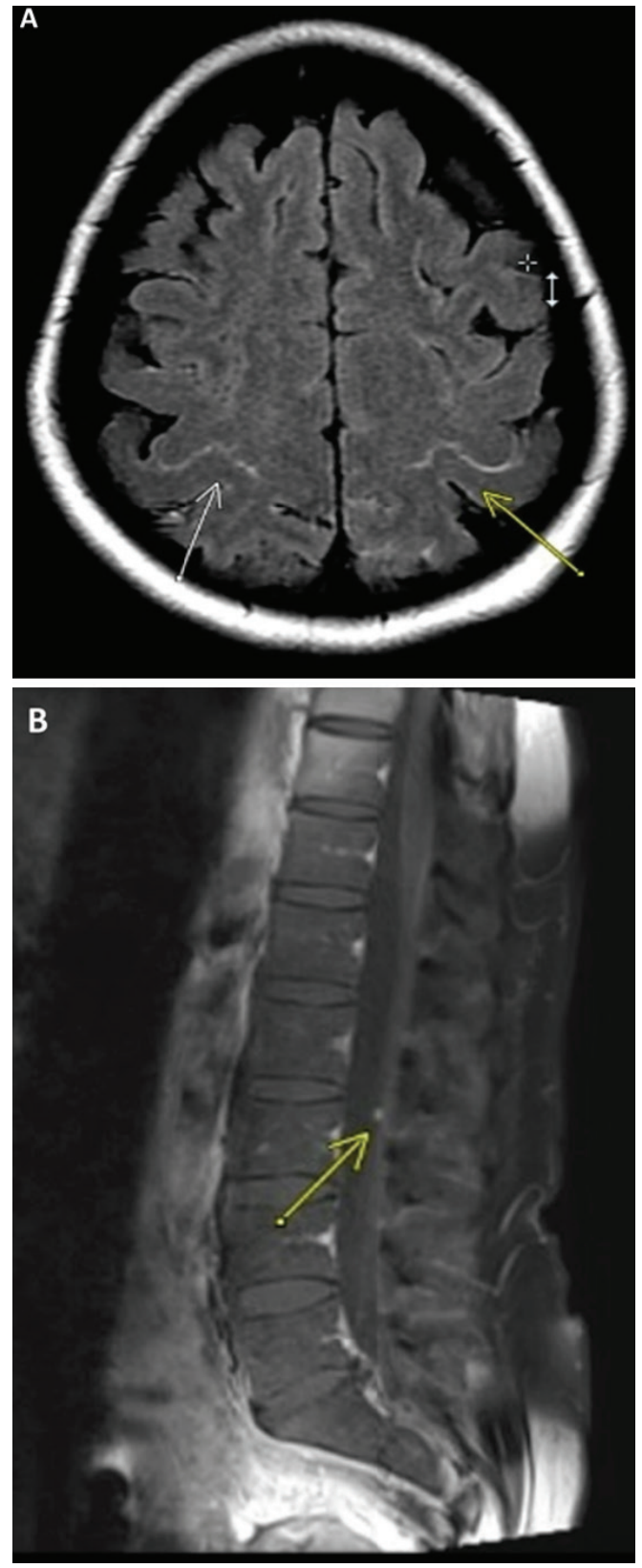

Figure 2. a) MRI brain with leptomeningal enhancement in the parietal sulci b) A leptomeningal enhancing focus along a nerve root in the lumbar spine.

MRI: Magnetic resonance imaging

systemic chemotherapy agent. The most common addition was radiotherapy in 30 patients (42\%). The most likely new agent was the addition of capecitabine in six patients (8\%). Twenty-eight (18\%) patients received intrathecal chemotherapy with 27 (96\%) receiving liposomal cytarabine and one (4\%) receiving thiotepa. The median OS for patients with no new treatment after LMC diagnosis was 0.7 months $(95 \% \mathrm{CI}: 0.6,1.2)$ and for those with a change in treatment after LMC diagnosis, 2.4 months (95\% CI: 1.6, 3.1) $(\mathrm{p}<0.001)$.

\section{Breast cancer subset analysis}

A separate analysis was performed specifically on the subset of patients with a primary breast cancer (see Table 1 for tumor 
characteristics). Thirty-seven breast cancer patients received radiotherapy for LMC (56\%) and 64 received chemotherapy for either the primary disease or LMC (97\%), with 22 patients (36\%) receiving intrathecal chemotherapy and 42 patients (64\%) receiving hormonal therapy.

Of the 66 patients, there were 64 (97\%) observed deaths; and the survival differed for patient based on their biomarker status. Median OS for all patients was 2.4 months (95\% CI: 1.2-4.4). Median OS for $\mathrm{ER}+/ \mathrm{PR}+/ \mathrm{HER} 2-$ patients $(\mathrm{n}=40,61 \%)$ was 4.1 months (CI: 1.7, 9.8), for triple negative breast cancer (TNBC) patients $(\mathrm{n}=17,26 \%)$ was 0.9 months (CI: $0.2,1.9)$ and for HER2+ patients $(n=6,9 \%)$ was 0.7 months (CI: $0.0,15.8$ ). A significant difference in OS between subtypes based on hormone receptor status was found ( $\mathrm{p}-0.002$, logrank test). OS was improved with new treatment after LMC diagnosis, with median OS of 2.8 months (CI: 1.3, 5.7) in treated patients ( $\mathrm{n}=$ $57,86 \%$ ) compared to 1.2 months (CI: $0.03,3.6$ ) in untreated patients $(\mathrm{n}=9,14 \%)(\mathrm{p}-0.026)$. The median OS in CSF negative patients was 15.3 months (CI: 3.6, 30.1), 6.9 months in CSF equivocal patients (CI: $1.5,76.2$ ), and 0.9 months in CSF positive patients (CI: 0.4, 2.0) ( $\mathrm{p}=0.009$, Log rank test).

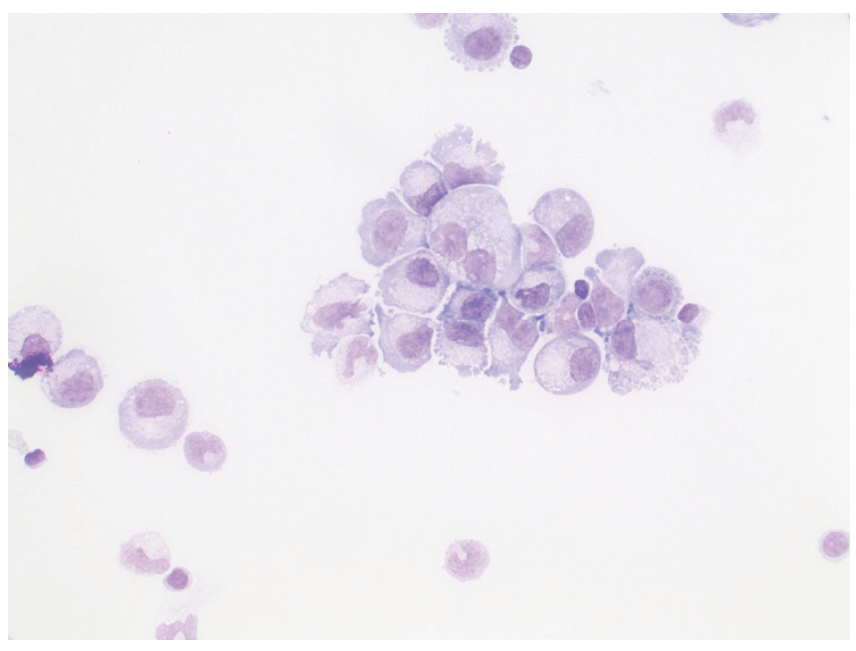

Figure 3. CSF cytology showing LMC from a patient with poorly differentiated gastric carcinoma with signet ring features

\section{CSF: Cerebrospinal fluid, LMC: Leptomeningeal carcinomatosis}

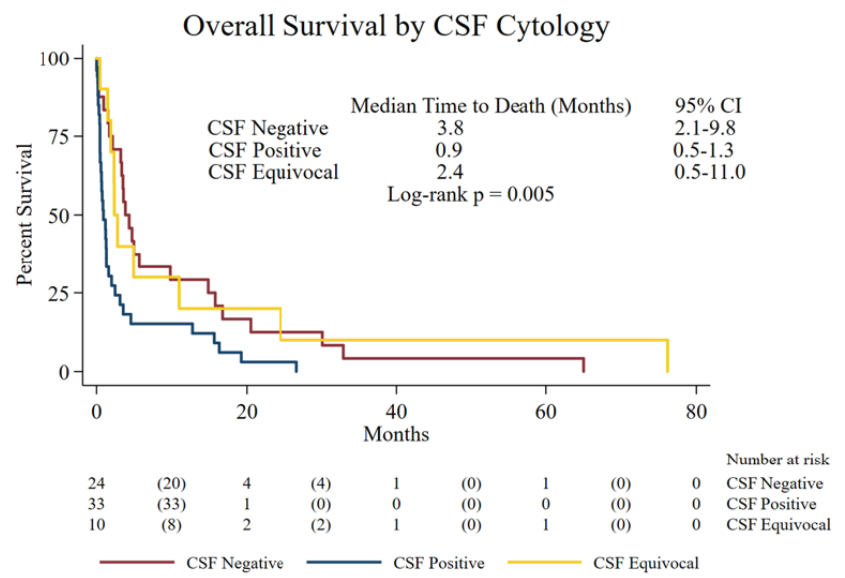

Figure 4. Kaplan-Meier survival curves showing the overall survival based on the CSF cytology

CSF: Cerebrospinal fluid

\section{Discussion:}

In patients with solid tumor malignancies, LMC is considered one of the most serious complications. We present a comprehensive overview of diagnostic methods and treatments of patients with LMC associated with solid tumors over a 10 -year period at our institution. LMC is commonly associated with breast cancer, lung cancer, skin melanoma along with various other cancers $(5,30)$. In our cohort, all patients underwent MRI of the brain and/or spine and $97 \%$ demonstrated radiographic evidence of LMC. This high rate demonstrates that at our institution MRI is the preferred initial diagnostic modality prior to attempting high volume LP.

The presence of malignant cells in the CSF versus equivocal or negative cytology was associated with a significantly lower overall survival in our cohort (0.9 months vs 3.8 months). This highlights the importance of repeating LP if CSF is initially negative as accurate CSF cytology is essential to further delineate an individual patient's prognosis.

Patients with LMC at our institution most commonly presented with stage IV breast cancer, lung cancer, or melanoma with metastases to the brain or bone. In the literature, the survival from the time of diagnosis of LMC is 4 to 6 weeks without treatment and 2 to 6 months with therapy $(5,6,22-25,31)$. Our cohort included 153 patients with a mixed population including patients who received treatment and some who proceeded with comfort care or hospice alone following diagnosis of LMC. The median OS of our cohort was 1.9 months (CI: 1.3, 2.5).

In our study, treatment of LMC either by intrathecal chemotherapy, radiation to the brain or spine, or systemic therapy was associated with an improvement in survival versus no treatment (Figure 4). The higher CSF protein level present in patients with LMC demonstrates that there is likely a blood-brain barrier disruption and resultant increased levels of systemic chemotherapy delivered to the subarachnoid space (32). Systemic chemotherapy is primarily based on the histology of the primary tumor as in other forms of metastatic disease. Use of systemic cytotoxic agents such as high-dose methotrexate can induce a response in LMC from various solid tumors and improve survival outcomes, however its use is limited due to systemic side effects, the potential for significant hematologic toxicity and the need for inpatient administration (32). A significant limitation to the efficacy of systemic chemotherapy in the treatment of LMC is resistance to therapy as most patients developed disease progression despite multiple lines of systemic chemo and/or hormonal therapy prior to development of LMC.

Intrathecal methotrexate is a commonly utilized and relatively welltolerated agent associated with leukoencephalopathy (33). The efficacy of intrathecal trastuzumab is currently unclear and is being investigated for LMC from HER2-positive breast cancer given that systemic trastuzumab appears to have poor penetration into the CSF $(26,27)$. Liposomal cytarabine administered intrathecally has been associated with complete cytological remission likely due to its unique formulation which allows for persistence for up to 28 days in the CSF (19). However, this agent is no longer available for clinical use due to the manufacturer discontinuing production of this preparation; the shorter acting version can still be utilized. The decision to use intrathecal chemotherapy in the setting of LMC must be carefully considered taking into account the extent and status of systemic disease, the patient's functional status, and impact of the treatment and frequency of administration on the quality of life. 
Breast cancer appears to be particularly responsive to therapy with overall survival of 7.5 months with therapy in the literature (34). However, as evidenced in our cohort of breast cancer patients, TNBC and HER2+ patients have a significantly worse prognosis as compared to $\mathrm{ER}+/ \mathrm{PR}+/ \mathrm{HER} 2-$ patients. Patients with a primary lung cancer or melanoma appear to be less responsive. In these patients, targeted therapy in the setting of certain actionable mutations (e.g osimertinib in EGFR mutant NSCLC or BRAF inhibitor or checkpoint inhibitors in melanoma) have shown preliminary evidence of activity against LMC in these tumors (35). In this mixed cohort of patients with and without treatment, the median OS for primary breast cancer was 2.4 months which was significantly longer than primary lung cancer (OS: 1.3 months) and primary melanoma (OS: 1.7 months). Despite treatment, prognosis remains poor and confirmation of diagnosis is challenging.

\section{Strengths and limitations}

A strength of the study was the relatively large cohort size of 153 patients given the relative rarity of LMC. We used not only the ICD9 and ICD10 codes for carcinomatous meningitis or unspecified meningeal disease, but we also included patients who were diagnosed with a malignant solid tumor, who had undergone a procedure indicative of leptomeningeal carcinomatosis according to CPT codes. There are several limitations to our study including its retrospective nature, somewhat limited sample size for specific treatment modalities, and the 5-year period of review during which time imaging techniques and treatment options changed significantly for many solid tumors. The range of treatments and histologic diagnoses was too heterogeneous, and sample sizes were too small to statistically assess the impact of specific drugs or treatment modalities on specific cancer diagnoses. Future multiinstitution studies may reveal more information specific to LMC of difference histologies.

In conclusion, the risks and benefits of treatment in patients with LMC must be considered in detail on an individual basis. This study may provide additional information for physicians to communicate prognostic information to patients based on an individual's cancer type, stage, grade, molecular status, and CSF cytology results.

Ethics Committee Approval: This study was an IRB-approved (registration \#: OSU2016C0053) retrospective chart review of clinical and histopathologic data from patients treated at The Ohio State University Comprehensive Cancer Center, (OSUCCC)-James that was initially approved on 05/04/2016 between January $1^{\text {st }}, 2010$ and December $31^{\text {st }}, 2015$.

Informed Consent: Retrospective study.

Peer-review: Externally peer-reviewed.

\section{Author Contributions}

Conception: H.R., M.K., E.M., A.S., A.G.; Design: H.R., M.K., E.M., A.S., A.G.; Data Collection and/or Processing: H.R., M.K., E.M., M.D.P.G.P.E., A.M., I.A.; Analysis and/or Interpretation: M.P., J.A.S., M.L., R.W., S.S., D.S., J.V., M.C., P.G., V.K.P., N.W., B.R., A.M.N.; Writing: H.R., M.K.; Critical Review: M.L., R.W., S.S., D.S., J.V., M.C., P.G., V.K.P., N.W., B.R., A.M.N.

Conflict of Interest: No conflict of interest was declared by the authors.

Financial Disclosure: The authors declare that this study received no financial

\section{References}

1. Nugent JL, Bunn PA Jr, Matthews MJ, Ihde DC, Cohen MH, Gazdar A, et al. CNS metastases in small cell bronchogenic carcinoma: increasing frequency and changing pattern with lengthening survival. Cancer 1979; 44: 1885-1893. (PMID: 227582) [CrossRef]

2. Boyle R, Thomas M, Adams JH. Diffuse involvement of the leptomeninges by tumour--a clinical and pathological study of 63 cases. Postgrad Med J 1980; 56: 149-158. (PMID: 7393804) [CrossRef]

3. Yung WA, Horten BC, Shapiro WR. Meningeal gliomatosis: a review of 12 cases. Ann Neurol 1980; 8: 605-608. (PMID: 6260012) [CrossRef]

4. Price RA, Johnson WW. The central nervous system in childhood leukemia. I. The arachnoid. Cancer 1973; 31: 520-533. (PMID: 4511909) [CrossRef]

5. Groves MD. New strategies in the management of leptomeningeal metastases. Arch Neurol 2010; 67: 305-312. (PMID: 20212228) [CrossRef]

6. Freilich RJ, Krol G, DeAngelis LM. Neuroimaging and cerebrospinal fluid cytology in the diagnosis of leptomeningeal metastasis. Ann Neurol 1995; 38: 51-57. (PMID: 7611725) [CrossRef]

7. Groves MD. Leptomeningeal disease. Neurosurg Clin N Am 2011; 22 : 67-78, vii. (PMID: 21109151) [CrossRef]

8. Yap HY, Yap BS, Tashima CK, DiStefano A, Blumenschein GR. Meningeal carcinomatosis in breast cancer. Cancer 1978; 42: 283-286. (PMID: 667799) [CrossRef]

9. Aroney RS, Dalley DN, Chan WK, Bell DR, Levi JA. Meningeal carcinomatosis in small cell carcinoma of the lung. Am J Med 1981; 71 : 26-32. (PMID: 6264785) [CrossRef]

10. Bruna J, Simó M, Velasco R. Leptomeningeal metastases. Curr Treat Options Neurol 2012; 14: 402-415. (PMID: 22736147) [CrossRef]

11. Kesari S, Batchelor TT. Leptomeningeal metastases. Neurol Clin 2003; 21: 25-66. (PMID: 12690644) [CrossRef]

12. Grossman SA, Trump DL, Chen DC, Thompson G, Camargo EE. Cerebrospinal fluid flow abnormalities in patients with neoplastic meningitis. An evaluation using 111 indium-DTPA ventriculography. Am J Med 1982; 73: 641-647. (PMID: 6814249) [CrossRef]

13. Chamberlain MC, Corey-Bloom J. Leptomeningeal metastases: 111indium-DTPA CSF flow studies. Neurology 1991; 41: 1765-1769. (PMID: 1944906) [CrossRef]

14. Siegal T, Mildworf B, Stein D, Melamed E. Leptomeningeal metastases: reduction in regional cerebral blood flow and cognitive impairment. Ann Neurol 1985; 17: 100-102. (PMID: 3985577) [CrossRef]

15. Costa R, Kumthekar P. Management of central nervous system metastases in breast cancer. Breast 2018: 942-690. e7. [CrossRef]

16. Le Rhun E, Weller M, Brandsma D, Van den Bent M, de Azambuja E, Henriksson R, et al; EANO Executive Board and ESMO Guidelines Committee. EANO-ESMO Clinical Practice Guidelines for diagnosis, treatment and follow-up of patients with leptomeningeal metastasis from solid tumours. Ann Oncol 2017;28(Suppl 4):iv84-iv99. (PMID: 28881917) [CrossRef]

17. Yousem DM, Patrone PM, Grossman RI. Leptomeningeal metastases: MR evaluation. J Comput Assist Tomogr 1990; 14: 255-261. (PMID: 2312855) [CrossRef]

18. Chamberlain MC. Leptomeningeal metastasis. Semin Neurol 2010; 30 : 236-244. (PMID: 20577930) [CrossRef]

19. Bohn JP, Reinstadler V, Pall G, Stockhammer G, Steurer M, Oberacher $\mathrm{H}$, et al. Cerebrospinal fluid drug concentrations and clinical outcome of patients with neoplastic meningitis treated with liposomal cytarabine. Eur J Drug Metab Pharmacokinet 2019; 44: 845-851. (PMID: 31435852) [CrossRef] 


\section{Rinehardt et al. LMC: Diagnosis, Management and Outcomes}

20. Kaplan JG, DeSouza TG, Farkash A, Shafran B, Pack D, Rehman F, et al. Leptomeningeal metastases: comparison of clinical features and laboratory data of solid tumors, lymphomas and leukemias. J Neurooncol 1990; 9: 225-229. (PMID: 2086737) [CrossRef]

21. Chamberlain MC. Neoplastic meningitis. J Clin Oncol 2005; 23: $3605-$ 3613. Retraction in: J Clin Oncol 2010; 28: 4018. (PMID: 15908671) [CrossRef]

22. Clarke JL, Perez HR, Jacks LM, Panageas KS, Deangelis LM. Leptomeningeal metastases in the MRI era. Neurology 2010; 74: 14491454. (PMID: 20439847) [CrossRef]

23. Herrlinger U, Förschler H, Küker W, Meyermann R, Bamberg M, Dichgans J, et al. Leptomeningeal metastasis: survival and prognostic factors in 155 patients. J Neurol Sci 2004; 223: 167-178. (PMID: 15337619) [CrossRef]

24. Oechsle K, Lange-Brock V, Kruell A, Bokemeyer C, de Wit M. Prognostic factors and treatment options in patients with leptomeningeal metastases of different primary tumors: a retrospective analysis. J Cancer Res Clin Oncol 2010; 136: 1729-1735. (PMID: 20204406) [CrossRef]

25. Waki F, Ando M, Takashima A, Yonemori K, Nokihara H, Miyake M, et al. Prognostic factors and clinical outcomes in patients with leptomeningeal metastasis from solid tumors. J Neurooncol 2009; 93: 205-212. (PMID: 19043775) [CrossRef]

26. Mack F, Baumert BG, Schäfer N, Hattingen E, Scheffler B, Herrlinger U, et al. Therapy of leptomeningeal metastasis in solid tumors. Cancer Treat Rev 2016; 43: 83-91. (PMID: 26827696) [CrossRef]

27. Beauchesne P. Intrathecal chemotherapy for treatment of leptomeningeal dissemination of metastatic tumours. Lancet Oncol 2010; 11: 871-879. (PMID: 20598636)
28. Hermann B, Hültenschmidt B, Sautter-Bihl ML. Radiotherapy of the neuroaxis for palliative treatment of leptomeningeal carcinomatosis. Strahlenther Onkol 2001; 177: 195-199. (PMID: 11370554) [CrossRef]

29. Harris PA, Taylor R, Thielke R, Payne J, Gonzalez N, Conde JG. Research electronic data capture (REDCap)--a metadata-driven methodology and workflow process for providing translational research informatics support. J Biomed Inform 2009; 42: 377-381. (PMID: 18929686) [CrossRef]

30. Chamberlain MC. Leptomeningeal metastases: a review of evaluation and treatment. J Neurooncol 1998; 37: 271-284. (PMID: 9524085) [CrossRef]

31. Mayer RJ, Berkowitz RS, Griffiths CT. Central nervous system involvement by ovarian carcinoma: a complication of prolonged survivial with metastatic disease. Cancer 1978; 41: 776-783. (PMID: 630551) [CrossRef]

32. Scott BJ, Kesari S. Leptomeningeal metastases in breast cancer. Am J Cancer Res 2013; 3: 117-126. (PMID: 23593536) [CrossRef]

33. Zairi F, Kotecki N, Rodrigues I, Baranzelli M-C, Andre C, Dubois F, et al. Prospective follow-up of a cohort of 112 patients with leptomeningeal metastases of breast cancer recruited from 2007 to 2011: Prognostic factors. Am Soc Clin Oncol 2012; 30(Suppl): 2070. [CrossRef]

34. Chamberlain MC, Kormanik PR. Carcinomatous meningitis secondary to breast cancer: predictors of response to combined modality therapy. J Neurooncol 1997; 35: 55-64. (PMID: 9266441) [CrossRef]

35. Geukes Foppen MH, Brandsma D, Blank CU, van Thienen JV, Haanen JB, Boogerd W. Targeted treatment and immunotherapy in leptomeningeal metastases from melanoma. Ann Oncol 2016; 27: 11381142. (PMID: 26961150) [CrossRef] 